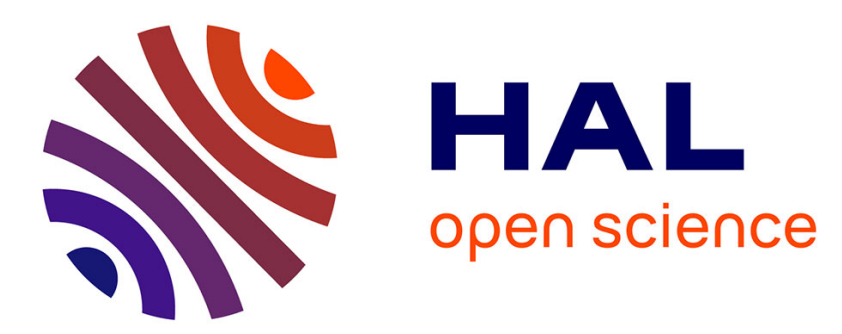

\title{
The roles of conduit redundancy and connectivity in xylem hydraulic functions
}

Assaad Mrad, Daniel M. Johnson, David M. Love, Jean-christophe Domec

\section{To cite this version:}

Assaad Mrad, Daniel M. Johnson, David M. Love, Jean-christophe Domec. The roles of conduit redundancy and connectivity in xylem hydraulic functions. New Phytologist, 2021, pp.1-12. 10.1111/nph.17429 . hal-03251444

\section{HAL Id: hal-03251444 \\ https://hal.inrae.fr/hal-03251444}

Submitted on 7 Jun 2021

HAL is a multi-disciplinary open access archive for the deposit and dissemination of scientific research documents, whether they are published or not. The documents may come from teaching and research institutions in France or abroad, or from public or private research centers.
L'archive ouverte pluridisciplinaire HAL, est destinée au dépôt et à la diffusion de documents scientifiques de niveau recherche, publiés ou non, émanant des établissements d'enseignement et de recherche français ou étrangers, des laboratoires publics ou privés.

\section{(ㄷ)(1) $\$$}

Distributed under a Creative Commons Attribution - NonCommerciall 4.0 International 


\title{
The roles of conduit redundancy and connectivity in xylem hydraulic functions
}

\author{
Assaad Mrad $^{1,2,3}$ (D), Daniel M. Johnson ${ }^{4}$ (D), David M. Love ${ }^{4}$ (D) and Jean-Christophe Domec ${ }^{1,5}$ (iD)

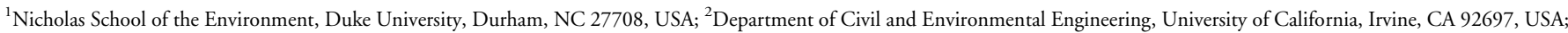 \\ ${ }^{3}$ Department of Engineering, Wake Forest University, Winston-Salem, NC 27101, USA; ${ }^{4}$ Warnell School of Forestry and Natural Resources, University of Georgia, Athens, GA 30602, \\ USA; ${ }^{5}$ Bordeaux Sciences Agro, UMR 1391 INRA-ISPA, Gradignan Cedex 33175, France
}

Author for correspondence:
Assaad Mrad
Email: mradassaad2@gmail.com

Received: 10 December 2020

Accepted: 20 April 2021

New Phytologist (2021)

doi: 10.1111/nph.17429

Key words: Acer (maples), connectivity, embolism spread, grouping, hydraulic conductance, xylem.

\section{Summary}

- Wood anatomical traits shape a xylem segment's hydraulic efficiency and resistance to embolism spread due to declining water potential. It has been known for decades that variations in conduit connectivity play a role in altering xylem hydraulics. However, evaluating the precise effect of conduit connectivity has been elusive. The objective here is to establish an analytical linkage between conduit connectivity and grouping and tissue-scale hydraulics.

- It is hypothesized that an increase in conduit connectivity brings improved resistance to embolism spread due to increased hydraulic pathway redundancy. However, an increase in conduit connectivity could also reduce resistance due to increased speed of embolism spread with respect to pressure. We elaborate on this trade-off using graph theory, percolation theory and computational modeling of xylem. The results are validated using anatomical measurements of Acer branch xylem.

- Considering only species with vessels, increases in connectivity improve resistance to embolism spread without negatively affecting hydraulic conductivity. The often measured grouping index fails to capture the totality of the effect of conduit connectivity on xylem hydraulics.

- Variations in xylem network characteristics, such as conduit connectivity, might explain why hypothesized trends among woody species, such as the 'safety-efficiency' trade-off hypothesis, are weaker than expected.

\section{Introduction}

Plant xylem supplies water from the soil pores to the leaves to compensate for the loss of water molecules from leaves to the atmosphere. As stomata open to uptake carbon dioxide molecules needed for photosynthesis, water molecules evaporate. The water experiences a phase transition in the leaf parenchyma and escapes as vapor through the guard cells to a desiccating atmosphere. For every water molecule lost from leaves, the entire column of water must be pulled upwards from the soil-root interface or from plant water storage to compensate for this water molecule loss. This makes the conveyance of water from roots to leaves through the xylem unique; it is entirely passive with minimal energy expenditures (no pumping) and operationally relies on water loss from leaves via transpiration only (Venturas et al., 2017). However, the drawback of such a passive system is that water in the xylem is in a metastable state where cohesive forces between water molecules and adhesive forces between the molecules and the cell walls sustain large tensile stresses (Dixon \& Joly, 1895). This tension is then transmitted to the xylem cell walls and sap.

The xylem tissue bears a complex network of sap-transporting conduits that form many parallel pathways for water movement. The network of conduits, extending from the roots to the leaves, is redundant such that if a conduit is damaged, many other pathways still exist for the water to reach the leaves. The water is able to move from one conduit to the next through interconduit pits (hereafter 'pits'), which are openings in the secondary cell wall of conduits that allow lateral water transport. Pathway redundancy in xylem is necessary because conduits are often at the risk of dysfunction through gas bubble expansion.

The consequence of having sap under tension in a conduit is that some gas-filled bubbles can grow and fill the whole conduit. Embolisms inside conduits have been hypothesized to originate and spread through homogeneous nucleation, heterogeneous nucleation from a crack or impurities, or air-seeding from porous pits on conduit walls (Tyree et al., 1994). In the angiosperm clade, of main interest in this study, the pits contain a porous membrane, termed the pit membrane, that links conduits to each other. Pressure chamber experiments have lent support to the hypothesis of air-seeding via pores in the interconduit pit membranes by demonstrating the equivalence between a drop in sap pressure and a rise in the pressure of embolism contents (Cochard et al., 1992; Sperry \& Saliendra, 1994). This is because nucleation, or cavitation, requires negative water pressures to occur whereas air-seeding is dependent on the absolute value of the pressure difference between the gas in embolized vessels and the 
sap $(P)$. Air-seeding from adjacent conduits is considered the sole source of embolisms in this study. In other words, when a conduit is embolized, or air-filled, there is risk for embolism to spread to adjacent, connected conduits (Sperry \& Tyree, 1988). This requires an initial embolism event as exposure of the xylem sap to air due to disturbances such as fire and strong winds, herbivores or pathogens (Venturas et al., 2017) or de novo heterogenous nucleation. These initial embolisms might then spread to a larger number of vessels through air-seeding.

The ultimate impact of vessel embolism is related to its effect on organ and whole-plant hydraulic function. When a functional vessel is air-filled, at least one hydraulic pathway from segment inlet to outlet is lost, affecting whole-segment hydraulics. The way vessels are distributed and connected to one another determines the degree to which an embolism affects segment-level resistance to embolism spread. One such measure is the vulnerability to embolism curve (VC): a plot of the percentage loss of hydraulic conductivity (PLC) of a whole-segment against $P$ (Tyree \& Zimmermann, 2002). As $P$ increases, PLC increases from $0 \%$ to $100 \%$. The shape and location of the $\mathrm{VC}$ along the $P$ axis depends on embolism spread within the xylem network of that segment, the ease of which is determined by pit membrane ultrastructural properties and frequency.

However, being a 'macroscale' measure, the VC integrates network mechanisms beyond vessel to vessel embolism exchange. Examples of these mechanisms are the redundancy of the xylem hydraulic pathway (Ewers et al., 2007), the variability of conduit wall susceptibility to embolism spread along the segment crosssection (Venturas et al., 2019), patterns of disease epidemics on graphs (Roth-Nebelsick, 2019) and the so-called percolation threshold. The percolation threshold is an effective 'seal' against uninhibited embolism spread born out of vessel connectivity statistics (Callaway et al., 2000). As yet, the percolation threshold has not been utilized in the literature to infer VCs. It is a network property that links the speed of disease, or embolism, spread in a network based on the connectivity (number of neighbors) of the agents (vessels). It is these 'midscale' mechanisms, straddling the 'microscale' processes and 'macroscale' measures, that are of interest and frame the scope of the work here.

The basic property that controls xylem pathway redundancy and embolism 'percolation' is the average vessel connectivity $(\langle c\rangle)$. The average vessel connectivity in a xylem segment is the number of vessel neighbors (i.e. with a common contact wall providing hydraulic connection), averaged over all vessels in the xylem segment (Loepfe et al., 2007; Martínez-Vilalta et al., 2012; Newman, 2018). Unfortunately, $\langle c\rangle$ has rarely been reported in the literature but the grouping index (GI) is a measure commonly quantified by anatomists (Carlquist, 1984). The GI is the number of vessels in a xylem cross-section divided by the number of vessel groups. Therefore, the GI is a two-dimensional proxy to $\langle c\rangle$. To make use of the GI and link it to $\langle c\rangle$, we focus on a particular type of wood.

Our interest here is in angiosperm species that lack vasicentric tracheids (Carlquist, 1984; Pratt \& Jacobsen, 2018). Flowering plants with vasicentric tracheids can achieve pathway redundancy by surrounding vessels by tracheids (Hacke et al., 2009; Pratt et al., 2015). While vasicentric tracheids are dramatically smaller in diameter and length, they could be numerous enough to sustain significant 'back-up' flow in case an adjacent vessel is embolized (Sano et al., 2011). When anatomists report measures of vessel grouping such as the GI, they only count vessels and disregard tracheids. Therefore, by focusing on species lacking vasicentric tracheids, we purposely take advantage of data representing the true connectivity of the xylem hydraulic pathway. In particular, we will be utilizing data on seven species of the genus Acer (Lens et al., 2011) which belongs to the family Sapindaceae, generally lacking vasicentric tracheids (Carlquist, 1984).

The plant physiology literature has made significant strides in understanding the linkages between vessel (Hacke et al., 2006; Christman et al., 2009, 2012) and pit anatomy (Choat et al., 2008; Jansen et al., 2009; Lens et al., 2011; Li et al., 2016), vessel to vessel air-seeding, and whole segment resistance to embolism spread through the VC. However, the processes mediated by xylem network properties (Jacobsen \& Pratt, 2018) have received relatively less attention and remain elusive because pertinent measurements are lacking. Xylem network theory (Ewers et al., 2007) and modeling (Loepfe et al., 2007; Martínez-Vilalta et al., 2012; Mrad et al., 2018) provide viable avenues for investigating these processes. As long as the effect of the xylem network is unclear, opportunities to link anatomy and segment hydraulics and to generalize trends among plant species will be missed. How the xylem network and vessel connectivity affect whole-segment hydraulics and resistance to embolism spread is investigated here through a synergistic combination of three-dimensional (3D) xylem computer modeling and graph theory.

In what follows, we outline the modeling and theory employed and compare the analytical linkage between average vessel connectivity and xylem hydraulics to anatomical measurements of Acer xylem. We present an extension to a xylem hydraulic model (Mrad et al., 2018) to simulate maple branch xylem segments by matching certain measurements of xylem network structure. With the aid of the model and graph theory, we link xylem vessel anatomy and connectivity to xylem VC measures and hydraulic conductivity. Ultimately, we highlight that increasing vessel connectivity increases the magnitude of the 'air-entry' pressure of xylem segments, without compromising hydraulic efficiency. Then, we put these results in the context of maple xylem and the 'safety-efficiency' trade-off hypothesis.

\section{Description}

The 2D model presented in Mrad et al. (2018) is extended by introducing a $3 \mathrm{D}$ representation. The radial dimension is added to the model such that $3 \mathrm{D}$ growth ring sections are simulated. In its new form, the model is suited for diffuse-porous angiosperm xylem where conductive, vasicentric tracheids are absent or play a minimal role in total tissue hydraulics. In the following, the anatomical elements represented in the model are only briefly described because of significant overlap with the description in Mrad et al. (2018). By contrast, aspects of the model related to the $3 \mathrm{D}$ representation of xylem networks are extensively explained. 
Throughout, water is assumed to be incompressible and its properties, including viscosity and density, are constant and defined at $25^{\circ} \mathrm{C}$ and standard pressure. Water flow in all vessels is assumed to be laminar. Each vessel is represented by a vertical cylinder with diameter $D_{\mathrm{v}}$ and length $L_{\mathrm{v}}$. The vessel lumens are hydraulically connected by intervessel connections (IVCs). The IVCs link adjacent vessel elements along the radial or tangential directions. Every vessel has a specified contact wall area determined by the vessel contact fraction $\left(f_{\mathrm{c}}\right)$ and the vessel cylindrical wall area $\left(A_{\mathrm{v}}\right)$. The ensemble of all IVCs between two vessels constitute their contact wall. The contact wall is divided into a pitfield area using the pit-field area fraction $\left(f_{\mathrm{p} f}\right)$ such that the pitfield area of each vessel equals $A_{\mathrm{v}} \times f_{\mathrm{c}} \times f_{\mathrm{pf}}$. The pit-field area of each contact wall contains the pits and its size determines the number of pit membranes it contains. Our interest here is in the average of vessel or pit properties throughout a xylem segment denoted using the 〈property $\rangle$ notation. The aforementioned anatomical properties have been measured on seven Acer species in Lens et al. (2011).

\section{Modeling xylem networks: sap flow}

To calculate the sap flow across a xylem segment, it is necessary to determine the flow through vessel elements and IVCs. Water flow through vessel lumens is described by the Hagen-Poiseuille equation while sap flow through IVCs is described by a superposition of Sampson and Hagen-Poiseuille flow resistances (Supporting Information Methods S1).

The $k_{x a, \max }$ of a modeled xylem segment is then calculated. $k_{x a, \max }$ is the maximum xylem area-normalized hydraulic conductivity of a segment. $k_{x a \text {, max }}$ is estimated by solving an equivalent hydraulic resistor network subject to a pressure difference between its ends (Methods S1). The resistances of the vessel elements and IVCs follow the equations above. One end of the segment is set at a given pressure, say atmospheric pressure, while the other end is subject to a higher pressure to form a pressure difference $\Delta P_{\text {seg }}$. Then, we establish a set of linear equations to be solved simultaneously like an electric resistor circuit (Mrad et al., 2018). The set of equations consist of the Hagen-Poiseuille and Sampson flow equations mentioned in the previous paragraph for every vessel element and IVC (Methods S1). The solution of the set gives the sap flow through segment $Q_{\mathrm{seg}}$. The hydraulic conductance of the segment $\left(K_{\text {seg }}\right)$ is then estimated as:

$K_{\mathrm{seg}}=\frac{Q_{\mathrm{seg}}}{\Delta P_{\mathrm{seg}}}$.

Eqn 1

Additionally, $k_{x a, \max }$ is:

$k_{x a, \max }=\rho \frac{L_{\text {seg }}}{A_{\text {seg }}} K_{\text {seg }}$,

Eqn 2

where $L_{\text {seg }}$ and $A_{\text {seg }}$ are respectively the axial length of the simulated segment and its transverse area while $\rho$ is the density of pure water.

\section{Modeling xylem networks: embolism spread}

Air-seeding requires embolism spread to originate from an adjacent vessel through one of the pits connecting two vessels (Tyree \& Zimmermann, 2002). An embolism spreads to an adjacent vessel when the absolute value of the pressure difference between the air inside the embolism and the surrounding sap $(P)$ exceeds the capillary pressure (i.e. the air-seeding pressure (ASP) of the contact wall connecting two vessels).

In angiosperms, pit membrane anatomy and frequency throughout vessel wall surfaces determine the critical $P$ at which airseeding occurs. By restricting the gas bubble meniscus to sizes of the order of nanometers, pit membranes reduce the chance that the bubble will spread from the embolized vessel and fill the functional one. The growth of the bubbles is dictated by their size, the number of gas molecules in them and their surface chemistry (Schenk et al., 2017; Konrad et al., 2019; Kanduč et al., 2020). However, analytical relations linking pit structural properties and their function in blocking air-seeding are lacking. Difficulties encompass the formation of lipid-coated nanobubbles that allow for stable bubbles to exist in functional vessels (Schenk et al., 2015, 2017) and the presence of a rare and leaky pit (Christman et al., 2009). Measurements of pit membrane structure are thought to suffer from artifacts introduced through sample preparation and dehydration (Li et al., 2016). Moreover, the exact link between pit membrane anatomy and function is not yet complete due to the complex interactions between its 3D structure and existing chemical compounds (Kaack et al., 2019; Zhang et al., 2020).

Therefore, the effects of these pit traits on resistance to embolism spread is surrogated to a pit ASP distribution. This distribution surrogates the complexity of the pit membrane ultrastructure including thickness, pore size, quantity and chemistry. The pit membrane ASPs are sampled from a two-parameter Weibull distribution that differs among species (Christman et al. 2009). This distribution is given by (Christman et al., 2009):

$F_{m}(\mathrm{ASP})=1-\exp \left[-\left(\frac{\mathrm{ASP}}{a}\right)^{b}\right]$,

Eqn 3

where $a$ and $b$ are distribution parameters and $F_{m}$ (ASP) is the cumulative distribution function of pit membrane ASPs.

Embolisms spread through the leakiest membrane between two vessels (Christman et al., 2009). To account for this 'extreme-value' effect, the cumulative distribution function of ASP for a contact wall containing $N_{m}$ pit membranes is derived from an extreme-value distribution as (Mrad et al., 2018):

$$
F_{c}(\mathrm{ASP})=1-\left[1-F_{m}(\mathrm{ASP})\right]^{N_{m}}=1-\exp \left[-\left(\frac{\mathrm{ASP}}{a / N_{m}^{1 / b}}\right)^{b}\right] .
$$

Eqn 4

In other words, $F_{c}(\mathrm{ASP})$ is the probability that a given $P$ will exceed the ASP of a randomly chosen contact wall in the xylem network. Therefore, larger contact walls in the same xylem 
segment are more likely to have a leakier pit. Air-seeding is assumed to result in unstable bubbles that fill up the 'infected' vessel completely and instantaneously, rendering it nonfunctional. This simplification is plausible as unstable bubbles expand rapidly (in microseconds; Konrad \& Roth-Nebelsick, 2003; Hölttä et al., 2007). Such an idealization also eliminates the need to represent the aerodynamics of air expansion within a vessel and any concomitant interaction with water movement.

Having assigned each contact wall an ASP, the VC of the simulated growth ring section can be computed. An embolism is injected into random vessels inside the network. Then, the pressure of its contents is increased at successive steps while the water pressure is maintained at $P_{\text {atm }}$ akin to the air-injection technique (Salleo et al., 1992). After every increase in $P$, the initial random embolism could spread to other conduits based on the adjacent contact wall ASPs. At every step, with some conduits embolized and nonfunctional, the unsaturated xylem area-specific hydraulic conductivity $\left(k_{x a}\right)$ is computed. PLC is then determined as:

$\operatorname{PLC}(P)=100 \times\left(1-\frac{k_{x a}(P)}{k_{x a, \max }}\right)$.

Eqn 5

The VC is the curve plotting PLC against increasing $P$. For the ensuing analysis, $P_{50}$ is defined as the absolute value of pressure at which $\operatorname{PLC}\left(P_{50}\right)=50$. Similarly, $P_{88}$ and $P_{12}$ are defined at $\operatorname{PLC}\left(P_{88}\right)=88$ and $\operatorname{PLC}\left(P_{12}\right)=12$, respectively (Domec \& Gartner, 2001). In planta, water is under tension and therefore is under negative pressure. Here, we look at the absolute values of those pressures.

\section{Modeling xylem networks: implementation of Acer hydraulics and 3D structure}

To ensure the simulated xylem networks model real tissues, several anatomical and connectivity-related measurements parameterize the simulated segments as elaborated in this section The measured average vessel length $\left\langle L_{\mathrm{v}}\right\rangle$ and diameter $\left\langle D_{\mathrm{v}}\right\rangle, f_{\mathrm{c}}$ and $f_{\mathrm{pf}}$ for each species are used as inputs of the $3 \mathrm{D}$ version. These model inputs ensure that water flow resistance through vessels and the frequency of contact walls and pit-field areas are realistic. $\left\langle D_{\mathrm{v}}\right\rangle$ and $f_{\mathrm{pf}}$ are used to model the number of pit membranes per contact wall.

In addition to the $2 \mathrm{D}$ version, the $3 \mathrm{D}$ version of the model employs two measurements to realistically represent the connectivity and frequency of vessels in the segment. The GI equals the number of vessels in a xylem cross-section divided by the number of vessel groups, which also include solitary vessels (Carlquist, 1984), and the vessel frequency $\left(\mathrm{VA}_{x}^{-1}\right)$ equals the number of vessels per unit transverse xylem area (Fig. 1). The model employs both measures to tune the frequency of vessels in the 3D xylem segment, the probability of a radial IVC with an adjacent vessel, and the probability of a tangential IVC. The majority of IVCs in Acer are radial so we constrain the probability of tangential connections within $5 \%$ and $20 \%$. This interval is liberal and encompasses the estimated proportions of tangential connections of the Acer segments based on their cross-sectional images (Fig. 1). Model simulations show that the proportion of tangential connections alone is insufficient to explain variation in $k_{x a \text {, } \max }$ and $P_{50}$ among species (Fig. S1). Therefore, constraining the proportion of tangential connections to a liberal range will faithfully represent xylem conduit geometry while not reducing the generality of this study's conclusions. Because of this constraint, the probability of radial connections and the probability of vessel initiation are now determined using these two independent measurements: GI and $\mathrm{VA}_{x}^{-1}$ (Methods S2).

In Fig. 1, the xylem model ensures that vessel connectivity patterns in a modeled growth ring section match those in an Acer negundo section. The modeled ring cross-section shows vessel lumen of varying diameters (not represented) as colored dots. Those that are connected by an IVC have a red line connecting (a) Modeled ring

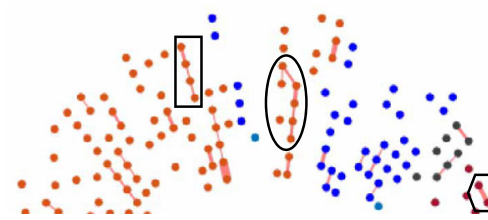

(b) Imaged ring

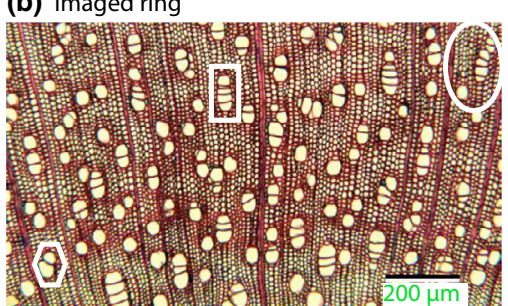

(c) Vulnerability curve comparison

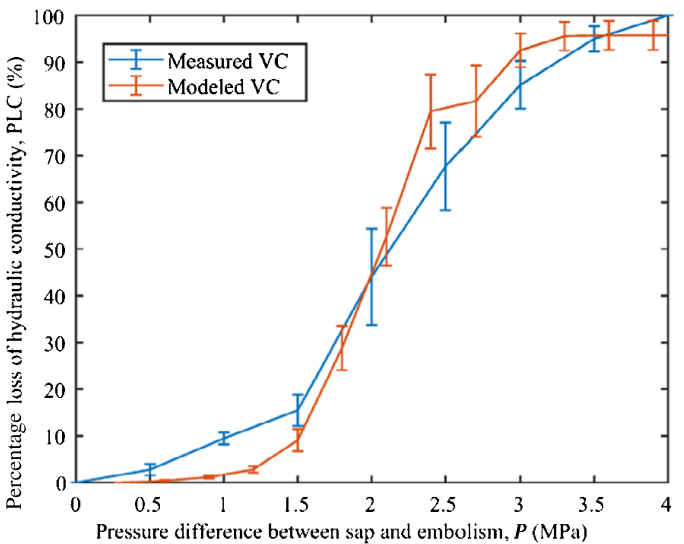

Fig. 1 Illustration showing the correspondence between (a) a cross-sectional view of a modeled Acer negundo ring and (b) an image of an A. negundo branch and (c) the resulting vulnerability to embolism curves (VC). The model does not represent each vessel in the image but approximates the averaged anatomy and xylem network properties. Three corresponding elements are highlighted by two rectangles, two ellipses and two hexagons. The rectangles show a uniquely radial file of vessels, the ellipses show a vessel group dominated by radial connections but with the occasional tangential or diagonal connection, and the hexagons show two vessels connected diagonally. Both the modeled and the imaged rings have a grouping index of 1.84 and a vessel density of $263 \mathrm{~mm}^{-2}$ (Lens et al., 2011). Similarly colored vessels are members of the same hydraulic component (see the Description section). The error bars around the measured and modeled VCs represent standard errors resulting from six A. negundo branches (blue) and 10 simulated branches (red). 
them (Fig. 1a). Vessels of the same color are hydraulically connected (Mrad et al., 2018). In the simulated segment, two vessels appearing disconnected in that transverse section does not preclude them being connected at another axial location.

Fig. 1(a) highlights three vessel groups showing correspondence to a group in an $A$. negundo imaged ring (Fig. 1b). The black and white rectangles show vessel groups with connections that are uniquely radial while the ellipses and hexagons have a single diagonal connection each. This highlights how the model recovers the preferential direction of vessel connections in the genus Acer.

In this paper, the pit membrane hydraulic parameters are fitted to obtain agreement with tissue-level hydraulic measurements because theoretical links between anatomy, hydraulic conductivity and resistance to embolism spread are uncertain, as explained above. For example, pore diameter is fit such that $k_{x a \text {, max }}$ of the whole xylem segment matches the measurements. Similarly, the two parameters, $a$ and $b$, of $F_{m}$ (ASP) (Eqns 3, 4) were tuned such that the modeled VC was similar to the measured VC from $P_{12}, P_{50}$ to $P_{88}$ (Fig. 1c). The match was not evaluated more rigorously because the results of this study do not depend on it.

\section{Theory}

Diffuse-porous species exemplified by Acer have quasiuniform anatomical properties from earlywood to latewood. As a result, only vessel connectivity is of interest here. The simulated xylem segments maintain stationary average anatomical traits throughout a growth ring.

Three-dimensional xylem is represented by a graph of nodes connected to each other via edges as in Fig. 2. We collapse each vessel onto a node, as shown in the correspondence between vessels in Fig. 2(a) and nodes in Fig. 2(b). The edges represent contact walls. A node has one of two states: functional or embolized. This representation of xylem is used to characterize embolism spread. Each edge in the graph representation is weighted by its ASP. As $P$ increases, it exceeds a higher fraction of edge ASPs (i.e. $F_{c}(P)$ is an increasing function of $P$ ) so more edges become conducive to embolism spread if one exists adjacently.

The number of vessels to which a given vessel is connected is called its connectivity $c$. For example, vessel 3 in Fig. 2 has $c_{3}=3$ and vessel 4 has $c_{4}=1$. Of main interest here is the average connectivity of the xylem network $\langle c\rangle$ over all its constituent vessels. The primary role of $\langle c\rangle$ in hydraulic pathway redundancy and extent of embolism propagation throughout the xylem network is now considered.

As alluded to above, at a given $P$ a fraction $F_{c}(P)$ of vessel to vessel edges are amenable to disease spread. In network or graph theory parlance, it is said that these edges are 'occupied'. Given these definitions, the process of embolism spread on xylem vessel networks falls under the realm of edge percolation processes on a graph (Callaway et al., 2000; Newman, 2018). Percolation processes stipulate a threshold fraction of 'occupied' edges $F_{c \text {,threshold }}$

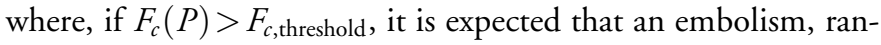
domly placed in the xylem network, will spread to the majority of the network. In other words, the percolation threshold is a limit on the fraction of contact walls with an ASP below the (a)

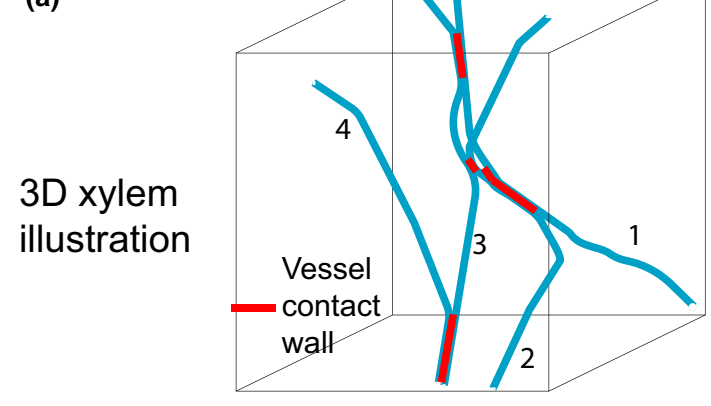

(b)

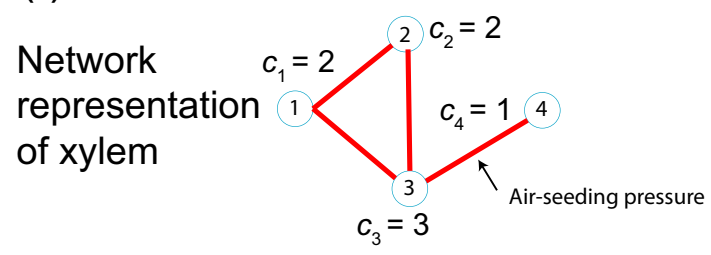

Fig. 2 Illustration of (a) a three-dimensional xylem network connected via contact walls, and (b) the connectivities $\left(c_{1}-c_{4}\right)$ of those conduits for embolism spread purposes. Every vessel (blue) in the xylem is collapsed onto a node while vessel contact walls (red) are represented by a bidirectional edge connecting the nodes. Every edge is weighted by the air-seeding pressure of the contact wall connecting the vessels. The length of each edge as drawn in (b) does not represent any property. The connectivity of every node in (b) is shown next to it. The average vessel connectivity of this example is $\langle c\rangle=2$.

pressure difference between sap and bubble contents $(P)$. When the threshold is exceeded, a randomly placed embolism is expected to spread to the majority of a xylem network. In a class of graphs applicable to xylem, called configuration models, the percolation threshold $F_{c \text {,threshold }}$ depends solely on the first $\langle c\rangle$ and second $\left\langle c^{2}\right\rangle$ moments of the vessel connectivity distribution:

$F_{c, \text { threshold }}=\frac{\langle c\rangle}{\left\langle c^{2}\right\rangle-\langle c\rangle}$.

Eqn 6

This result was first reported in a study of 'the resilience of the Internet to random breakdowns' (Cohen et al., 2000). Because percolation theory concerns graphs of infinite size and the xylem network is necessarily finite, the actual $F_{c \text {,threshold }}$ is expected to be smaller than its theoretical value (Eqn 6) due to 'finite-size' effects.

The direct consequence of vessel connectivity on a species' cavitation resistance is not straightforward and involves a trade-off explained in the Results. Determining the functional significance of vessel grouping is further complicated because pit structure (i.e. pit membrane thickness and pit chamber depth) and connectivity (i.e. GI and $\left\langle L_{\mathrm{v}}\right\rangle$ ) covary (Lens et al., 2011). Such covariations could lead to unexpected emergent tissue hydraulic behavior that is suited to a mechanistic exploration and which we address next.

\section{Varying vessel anatomy and connectivity}

Simulations were performed in which $\mathrm{VA}_{x}^{-1},\left\langle L_{\mathrm{v}}\right\rangle$ and $\left\langle D_{\mathrm{v}}\right\rangle$ are varied as well as the probabilities of radial and tangential 
connections to adjacent vessels. Such variations perturbed GI and $\langle c\rangle$. As these model properties were varied, others such as pit membrane diameter and the contact wall ASP distribution $F_{c}$ (ASP), which affect embolism spread resistance (Eqn 4), were kept constant. In other words, the distribution function's parameters $a$ and $b$ are varied such that $F_{c}$ (ASP) is independent of $N_{m}$ between simulations (Eqn 4). This allows us to disentangle the effect of vessel redundancy on vessel-to-vessel air-seeding and whole segment conductance and resistance to embolism spread. The objective of these simulations was to assess the role of vessel connectivity in overall segment safety, through $P_{12}, P_{50}$ and $P_{88}$, and hydraulic efficiency, through $k_{x a \text {, max }}$.

In the simulations discussed below, pit membrane pore diameter, $a$ and $b$ (Eqn 4) are fit to match Acer glabrum var. glabrum's $k_{x a, \max }$ and VC (Lens et al., 2011; Mrad et al., 2018, as in Fig. 1). However, the results are insensitive to the species used for initial fitting because they are presented in terms of normalized $k_{x a, \max }$ and PLC measures, as explained in what follows.

\section{Results}

When $\mathrm{VA}_{x}^{-1}$ in a segment cross-section increased, GI and $\langle c\rangle$ increased (Fig. S2). By contrast, the model indicated that $\left\langle D_{\mathrm{v}}\right\rangle$ and $\left\langle L_{\mathrm{v}}\right\rangle$ had no influence on measures of connectivity. The effect of $\mathrm{VA}_{x}^{-1}$ on $\langle c\rangle$ is due to conduit placement throughout a cross-section being random. A point pattern analysis of three Acer species supports this assumption (Martínez-Vilalta et al., 2012) and the anatomical measurements by Lens et al. (2011) show a strong correlation between $\mathrm{VA}_{x}^{-1}$ and GI, further corroborating this outcome. Conversely, while individual vessel length $L_{\mathrm{v}}$ is a strong predictor of the connectivity $c$ of a single vessel, $\left\langle L_{\mathrm{v}}\right\rangle$ in a xylem segment had no impact $\langle c\rangle$.

One of the hypothesized advantages of an increase in $\langle c\rangle$ is an increase in pathway redundancy, a measure of interest for many biological networks, including in the neurosciences and genetics (Tononi et al., 1999). As $\langle c\rangle$ increased from small values, redundancy increased significantly through the avoidance of vessel isolation. However, redundancy reached a maximum and saturated at and above a critical value of $\langle c\rangle$. To quantify the pathway redundancy of a simulated xylem, the fraction of embolized vessels at complete hydraulic failure (PLC $=100$ ) was used (Fig. 4a in orange). The rationale behind this metric is best explained through Fig. 3(a,b): the xylem network with lower $\langle c\rangle$ has a higher proportion of isolated conduits compared to the one with higher $\langle c\rangle$ even though the same conduits are embolized. It is then said that the xylem network in Fig. 3(b) is less redundant than the one in Fig. 3(a). Pathway redundancy is quantified by the fraction of embolized conduits at PLC $=100$ because it takes a larger fraction of embolized conduits for complete hydraulic failure (Fig. 3). Higher redundancy is achieved by reducing the instances of vessel isolation as a result of embolism spread events. That is achieved by increasing $\langle c\rangle$, which decreases the probability of a group of vessels becoming disconnected from the segment inlet or outlet. The maximum pathway redundancy $(c .90 \%)$ is achieved at $\langle c\rangle \approx 2.8$ on average and it stagnates with further increases in $\langle c\rangle$ (Fig. 4, top in orange). (a)

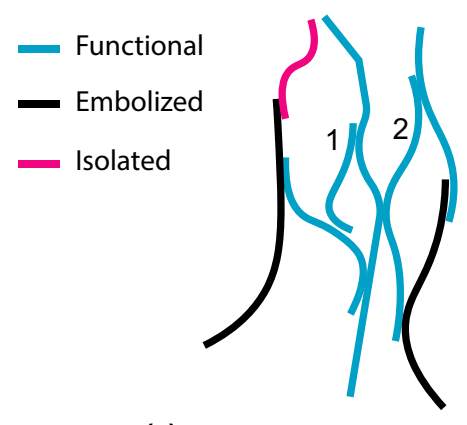

(c)

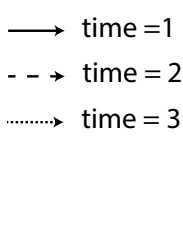

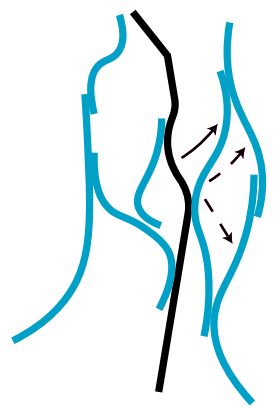

(d)

(b)
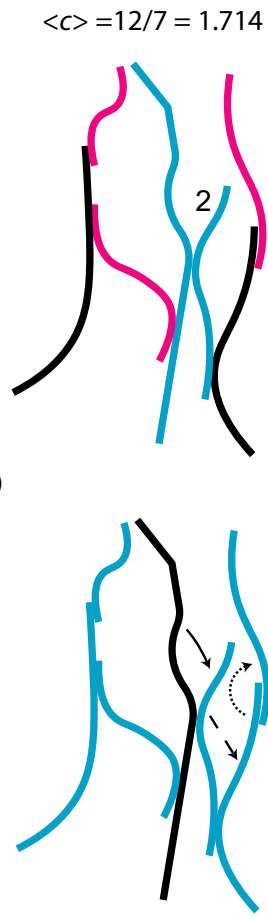

Fig. 3 Representation of how average conduit connectivity $\langle c\rangle$ affects redundancy $(a, b)$ and embolism spread speed $(c, d) .\langle c\rangle$ is calculated by counting the number of connections of each vessel and averaging. The xylem networks are similar for (a) and (c), and (b) and (d), respectively. The difference between the two networks is that the conduit labeled ' 1 ' is present in (a) and (c) but not in (b) and (d). The conduit labeled ' 2 ' is shorter in the latter panels and does not connect to the conduit on the top right. Panels (a) and (b) show how the network with lower $\langle c\rangle$ suffers from a greater number of isolated conduits than the network with higher $\langle c\rangle$. Conduit isolation occurs when a conduit still contains water but either the inlet or outlet is blocked by an embolized conduit. Panels (c) and (d) show how an embolism spreads faster in the network with higher $\langle c\rangle$ than the one with lower $\langle c\rangle$. The time step values in the legend are for illustration and are best understood as progressively increasing xylem water tension.

The hypothesized disadvantage of increased $\langle c\rangle$ is that it increases the speed of embolism spread with respect to $P$. $\langle c\rangle$ had minimal effect of spread speed until it reached a critical value. Above the critical value, the speed of embolism spread with respect to $P$ increased dramatically. The effect of $\langle c\rangle$ on embolism spread was represented by the fraction of embolized vessels at the average $P_{50}$ value $(\mathrm{PLC}=50)$ of all simulations combined. By determining the fraction of embolized vessels at this common $P$, we compared the extent of embolism spread at the same pressure occuring at the steepest portion of the ensemble-averaged VC. This represents the speed of embolism spread with respect to increasing $P$. By plotting the value of this metric against $\langle c\rangle$ per simulation, it is observed that the minimum spread rate occurs around $\langle c\rangle=3$ where c. $10 \%$, but as much as $20 \%$, of all vessels are embolized (Fig. $4 \mathrm{a}$ in blue).

The speed of embolism spread in xylem is tied by analogy to the so-called bond percolation process. By leveraging this conceptual link, it is deduced that spread speed increases with $\langle c\rangle$ above $\langle c\rangle=3$ because of a concomitant decrease in the percolation 

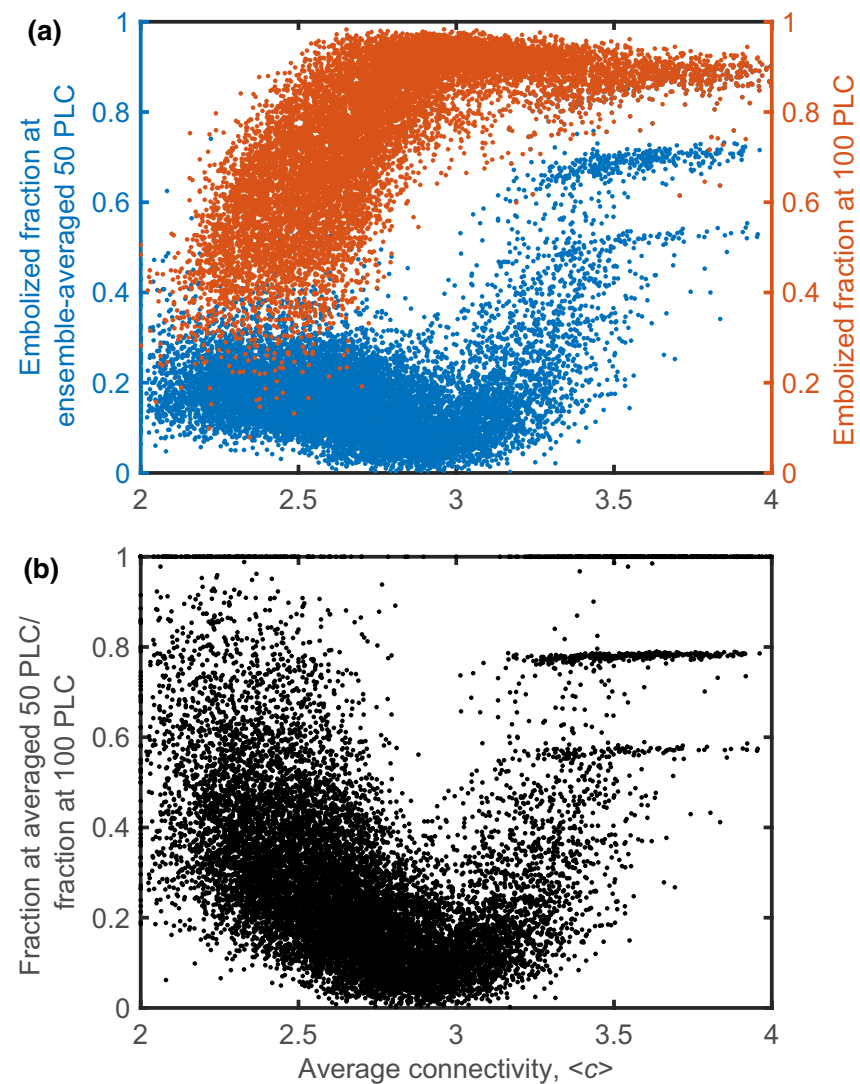

Fig. 4 The effect of average vessel connectivity $\langle c\rangle$ on pathway redundancy and embolism spread rate. (a) Embolism spread rate is quantified by the fraction of embolized vessels at the ensemble-averaged $50 \%$ loss of hydraulic conductivity (PLC; blue): average $P_{50}$ of all simulations combined. Pathway redundancy is quantified by the fraction of embolized vessels at 100 PLC of each simulation (orange). (b) Embolism spread rate divided by pathway redundancy highlighting an optimal $\langle c\rangle$ at which embolism spread is minimized.

threshold $\left(F_{c \text {,threshold }}\right)$. $F_{c \text {,threshold decreases from } c .70 \% \text { at }}$ $\langle c\rangle=2$ to $35 \%$ at $\langle c\rangle=3$ and further below as $\langle c\rangle$ increases (Fig.

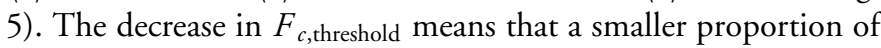
contact walls allowing embolism spread is required for a randomly placed embolism to propagate pervasively. This decline in $F_{c \text {,threshold }}$ explains the explosion in the fraction of embolized vessels above $\langle c\rangle=3$ (Fig. 4a). Above $\langle c\rangle=3, F_{c \text {,threshold declines }}$ below 35\% and the explosion in embolism spread speed occurs (Figs 4, 5).

The distance between the embolism spread rate and pathway redundancy represents the safety of hydraulic conductance to embolism spread. This distance is evaluated by taking the ratio of these two model outputs (Fig. 4b). This ratio is smallest when $\langle c\rangle$ is in the interval between 2.8 and 3 at $c .10 \%$. This analysis suggests that Acer species might conserve vessel connectivity, not GI (see below), to improve embolism resistance regardless of pit and vessel anatomy.

An increase in $\langle c\rangle$ to 3 increased $P_{12}$ significantly but not $P_{88}$, imparting a smaller increase to $P_{50}$ (Fig. 6). Above $\langle c\rangle$ to 3, $P_{12}, P_{50}$ and $P_{88}$ decreased such that the redundancy-spread speed trade-off was corroborated. The ordinates in Fig. 6 are

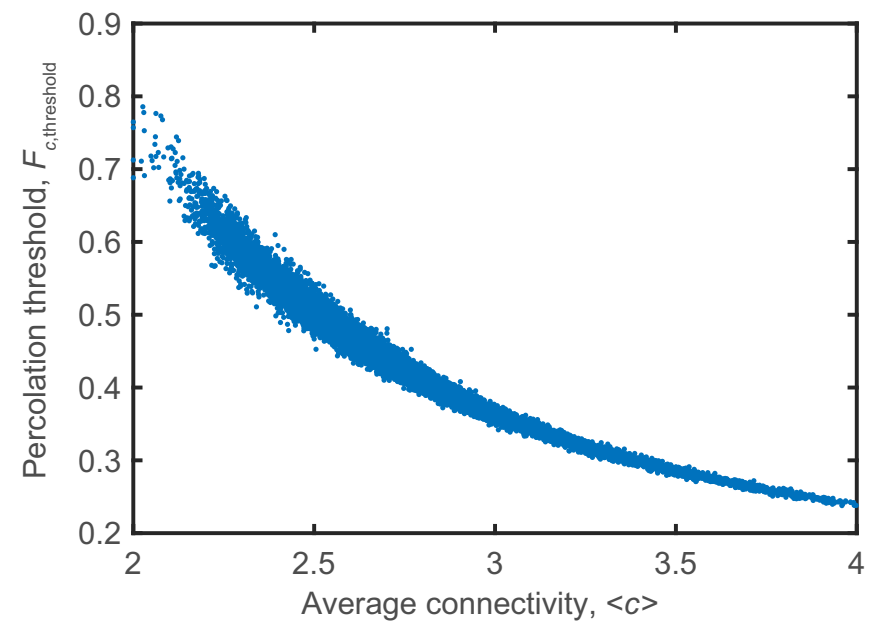

Fig. 5 The percolation threshold decreases with increasing average connectivity $\langle c\rangle$. The percolation threshold is a threshold fraction of conduit-to-conduit contact walls that allow an adjacent embolism to spread through them at a given xylem sap tension. If that threshold is exceeded, then a randomly placed embolism in the xylem network is expected to spread to the majority of the network.

normalized by their respective simulation means. This is to isolate the effects of $\langle c\rangle$ because the dimensional pressure values depend on pit membrane anatomy and frequency throughout vessel walls. An increase in $\langle c\rangle$ to 3 entailed an improvement in $P_{12}$, commonly referred to as the 'air-entry pressure'. In this case, variations in $P_{12}$ are not related to air-seeding and air entry dynamics but the concept of redundancy (Fig. 4). Above $\langle c\rangle=3$, gains in redundancy stagnated (Fig. 4) while the percolation threshold continued to decline (Fig. 5). The detrimental effects of a reduction in percolation threshold hold for $P_{50}$ and $P_{88}$ as well. By contrast, it appears from Fig. 6 that better resistance to embolism spread due to redundancy is strongest in the low-pressure portion of VCs (i.e. $P_{12}$ ). Therefore, the effect of $\langle c\rangle$ on $P_{88}$ is minimal below $\langle c\rangle=3$. As a result, increases in $P_{50}$ due to pathway redundancy were weaker compared to $P_{12}$ but still present.

In contrast to $\langle c\rangle$, the effect of GI on VC measures was weaker. The simulation scatterplots showed $P_{12}$ was significantly affected by GI whereas the relationship of GI to $P_{50}$ and $P_{88}$ was nuanced (Fig. 7). Having low GI (c. 1.5) did not preclude a high $P_{12}$ but a high GI constrains $P_{12}$ to higher values (Fig. $\left.7 \mathrm{a}\right)$. The Acer data conform to the scatter plot. The simulations show that $P_{88}$ and GI were uncorrelated for the simulations, leaving a weak relationship between GI and $P_{50}$ (Fig. $7 \mathrm{~b}, \mathrm{c}$ ).

$\left\langle L_{\mathrm{v}}\right\rangle$ is a strong predictor of maximum xylem area-specific hydraulic conductivity $\left(k_{x a, \max }\right.$; Fig. 8$)$ but not GI or $\left\langle D_{\mathrm{v}}\right\rangle$, and therefore the lumen fraction $F$. As was done for $P_{12}, P_{50}$ and $P_{88}, k_{x a, \max }$ is normalized by simulation and by Acer dataset means appropriately (Fig. 8). The model correlation between $\left\langle L_{\mathrm{v}}\right\rangle$ and $k_{x a, \max }$ indicates that water movement through vessel wall pits is the most restrictive to overall sap flow (Choat et al., 2008). With longer vessels, sap crosses a smaller number of pit membranes on average, thus increasing the conductivity of the 

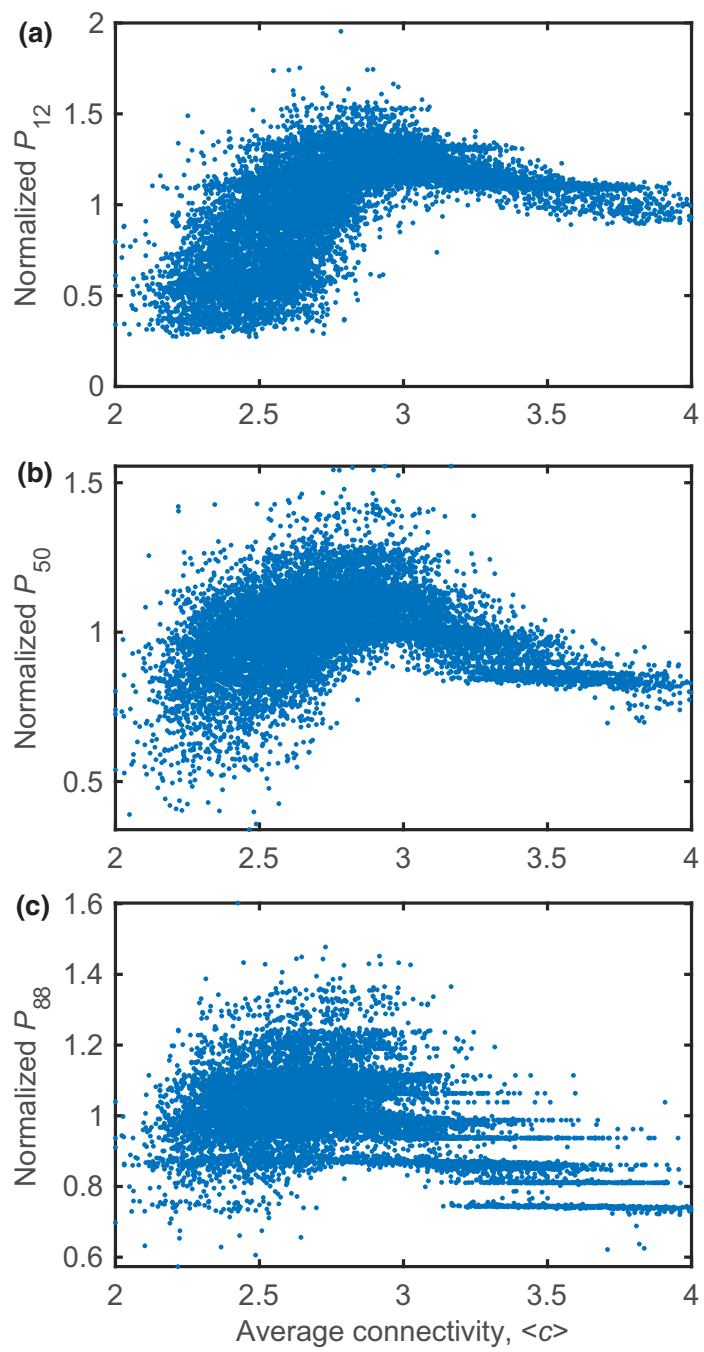

Fig. 6 Average vessel connectivity $\langle c\rangle$ affects (a) $P_{12}$ significantly, (b) $P_{50}$ mildly and (c) $P_{88}$ minimally. The ordinates are normalized by the respective simulation means. The dimensional pressure values are a function of pit membrane anatomy and the average vessel wall area occupied by pit membranes. Since interest here lies in the effect of $\langle c\rangle$ only, the ordinates are normalized.

whole segment (Fig. 8b). Over the ensemble of simulations, GI and $\left\langle D_{\mathrm{v}}\right\rangle$ were weakly correlated with $k_{x a \text {, max }}$ (Fig. 8a). In the Acer branches, GI and $\mathrm{VA}_{x}^{-1}$ are strongly correlated (Lens et al., 2011). Since $F$ in these branches is constrained to the range spanning 15-25\% (Fig. S3), there exists a trade-off between vessel frequency per transverse area $\left(\mathrm{VA}_{x}^{-1}\right)$ and $\left\langle D_{\mathrm{v}}\right\rangle$. This is because $F$ is the fraction of the transverse stem area occupied by vessel lumen $\left(F=\mathrm{VA}_{x}^{-1}(\pi / 4)\left\langle D_{\mathrm{v}}^{2}\right\rangle\right)$. As a result, it was hypothesized that increasing GI would lead to decreasing $k_{x a \text {, max }}$ due to a decrease in $\left\langle D_{\mathrm{v}}\right\rangle$ but this did not occur (Fig. 8a). The $\left\langle L_{\mathrm{v}}\right\rangle-k_{x a \text {, max }}$ relationship was minimally affected when $F$ was constrained in the model because $\left\langle L_{\mathrm{v}}\right\rangle$ had a stronger effect on $k_{x a, \max }$ than $\left\langle D_{\mathrm{v}}\right\rangle$ (gray scatterplots; Fig. $8 \mathrm{~b}$ ). Therefore, the presence of a correlation between GI and $k_{x a \text {, max }}$ in the Acer data (Lens et al., 2011; Fig. 8a) occurs because of a separate correlation between GI and $\left\langle L_{\mathrm{v}}\right\rangle$ (Lens et al., 2011).
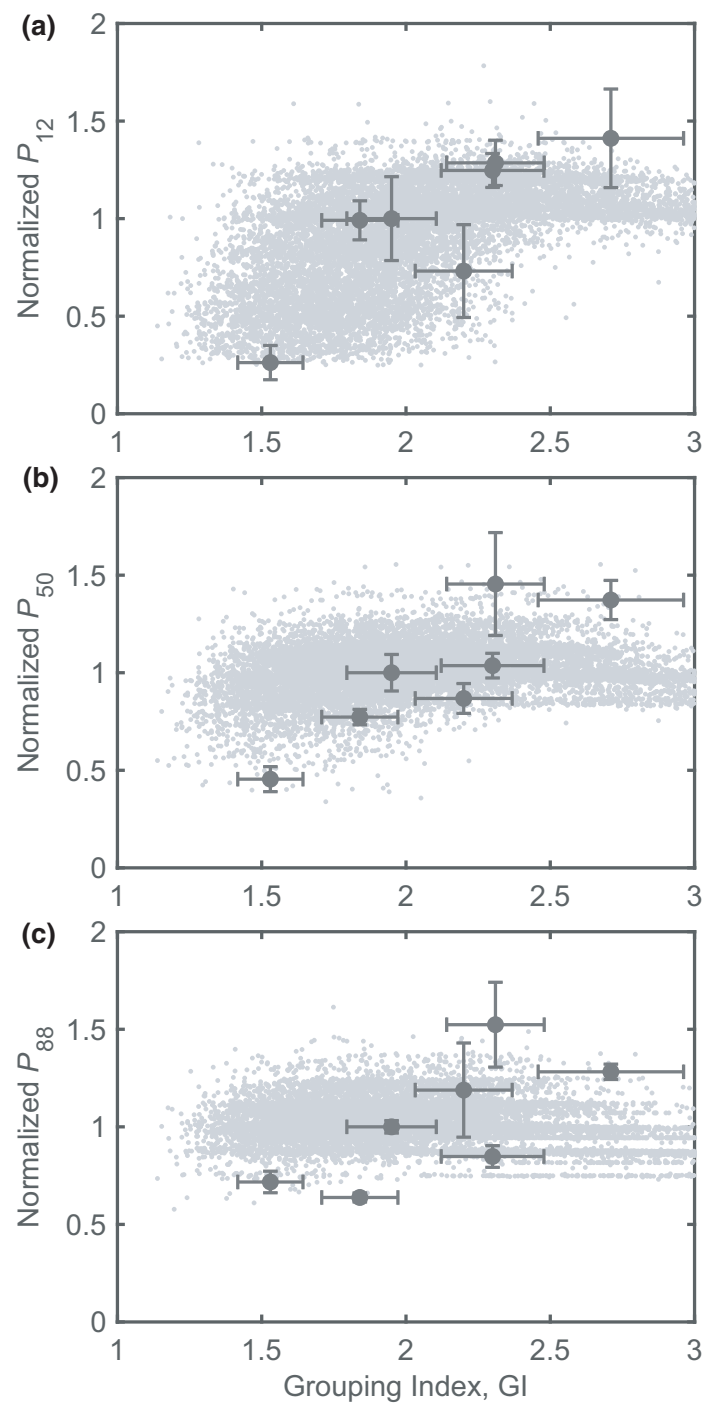

Fig. 7 The grouping index $(\mathrm{Gl})$ affects (a) $P_{12}$ significantly but its effect on (b) $P_{50}$ is nuanced while it bears no effect on (c) $P_{88}$. The ordinates are normalized by the respective simulation or Acer data means. The simulations are shown in light gray while data derived from Acer vulnerability to embolism curves are shown by large symbols. The error bars around the Acer data points are standard errors from Lens et al. (2011).

\section{Discussion}

The simulations suggested that vessel connectivity and grouping improved segment-level resistance to embolism spread without affecting hydraulic conductivity. Increases in $\langle c\rangle$ and GI lead to an increase in $P_{12}$ (more negative potential) through redundancy when all other anatomical features are held constant. There is a limit to the positive relationship between measures of vessel connection and $P_{12}$ at $\langle c\rangle=3$. At this value, embolism percolation dynamics overtook redundancy in influence but the Acer species did not seem to cross that limit. Increases in $\langle c\rangle$ below that limit increased $P_{12}$ more strongly than $P_{50}$ and $P_{88}$. As a result, higher vessel connectivity is thought to contribute to a steeper VC (Figs $6,7)$. 
(a)

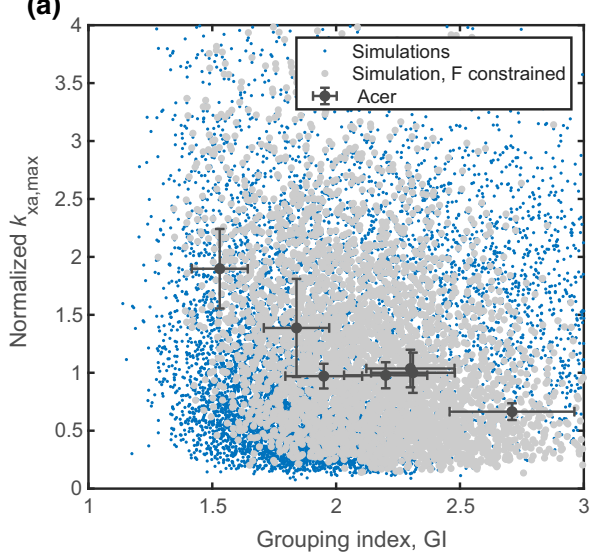

(b)

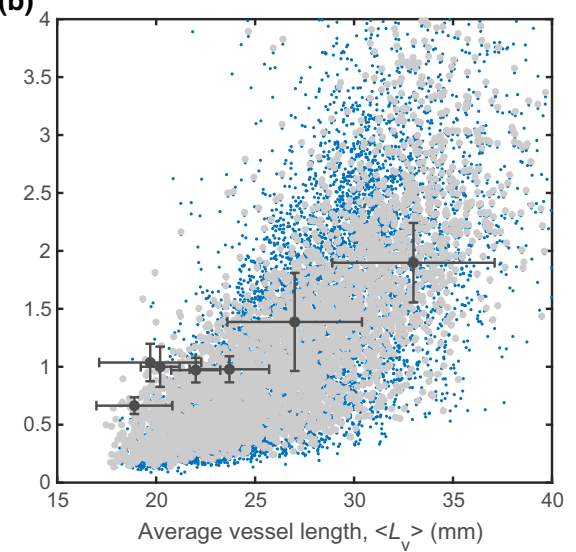

Fig. 8 While the Acer dataset (dark gray circles) shows significant correlations between the maximum xylem area-specific hydraulic conductivity ( $k_{x a}$, max) and (a) the grouping index $(\mathrm{Gl})$ and $(\mathrm{b})$ average vessel length $\left(\left\langle L_{v}\right\rangle\right)$, simulations show that only $\left\langle L_{v}\right\rangle$ is a significant predictor of $k_{x a \text {, max }}$ (blue and light gray circles). The simulations vary properties pertinent to vessel anatomy and connectivity, but not pit membrane hydraulic properties (see the Description section). Light gray circles are scatter plots of simulations where the vessel lumen fraction is constrained to between $15 \%$ and $25 \%$ (Fig. S3). The error bars around the Acer data points are standard errors from Lens et al. (2011). On the ordinate is $k_{x a \text {, max }}$, normalized appropriately by simulation or dataset means.

One could also use the relationship between $\langle c\rangle$ and redundancy to compare relative connectivities between species (Fig. 3 ). If one compares the proportion of air-filled conduits at a $\mathrm{PLC} \approx 100$ between different species, then one could infer that the species with the lowest proportion of embolized conduits is the least redundant and thus has lower $\langle c\rangle$. However, to make an inference about relative differences in $\langle c\rangle$ between species based on redundancy arguments, the species compared should all have only vessels as the main pathway for water and airseeding as the main mode of embolism spread in the xylem network.

Unfortunately, there does not exist data on Acer vessel connectivity in the literature due to the labor-intensive nature of these measurements. Consequently, we relied on a closely related measure, GI, which is reported for Acer species (Lens et al., 2011). GI is a $2 \mathrm{D}$ measure of vessel connection. Fig. 9 shows the relationship between the average transverse vessel connectivity $\left\langle c_{x}\right\rangle$ and GI. $\left\langle c_{x}\right\rangle$ quantifies only the connectivity apparent from a transverse stem section. So, it is the $2 \mathrm{D}$ equivalent of $\langle c\rangle .\left\langle c_{x}\right\rangle$ and GI differ if the fraction of tangential vessel connections varies from species to species as seen by comparing Fig. 9(b) and Fig. 9(c). While Fig. 9(b) and Fig. 9(c) have the same GI, the latter has a higher $\left\langle c_{x}\right\rangle$. Therefore, when vessel connections have a preferred direction (radial in Acer), they limit $\left\langle c_{x}\right\rangle$ and, therefore, $\langle c\rangle$ to lower values for the same GI. It is typical to have conduit connections in a predominantly preferred direction in angiosperm xylem with only vessels as the water pathway. One possible driver of this trend is the potential increase in the 'airentry' pressure of xylem $\left(P_{12}\right)$ due to increases in GI (Fig. 7a) while maintaining a high percolation threshold (Fig. 5) by keeping $\langle c\rangle$ to values low enough to prevent the explosion in the speed of embolism spread (Fig. 4). This supports the hypothesis that increasing GI is a necessary but insufficient condition to improve Acer resistance to embolism spread.
Nonetheless, trends between GI and PLC measures are similar, but weaker, to those with $\langle c\rangle$ despite the fact that we varied the fraction of tangential and diagonal connections four-fold in the simulations (Figs 7, S1). Most striking is the absence of a linear relationship between GI and $P_{50}$ but its presence in the Acer dataset (Lens et al., 2011). This suggests that the trend stems from the significant correlation between GI and another pit membrane property. This assertion is corroborated by a linear relationship between GI and $P_{88}$ in the dataset (Fig. 7b). Indeed, in the Acer data (Lens et al., 2011), $T_{\mathrm{m}}$ has been shown to be a strong predictor of resistance to embolism spread at the pit and vessel levels (Li et al., 2016) and at the xylem segment level (Lens et al., 2011). In a recent study on poplar, embolism resistance plasticity in Populus tremula $\times$ alba was shown to have a strong correlation between GI and $P_{50}$ (Lemaire et al., 2021). Concurrently, $P$. tremula $\times$ alba, a member of the family Salicaceae that largely does not possess vasicentric tracheids, has shown a significant decrease in the pit membrane area per vessel with an increase in $P_{50}$. These empirical examples support the hypothesis that GI is necessary for resistance to embolism spread in such angiosperm families but needs to be accompanied by variations in pit and vessel anatomy.

If increasing $\langle c\rangle$ leads to improved xylem resistance to embolism spread without negatively affecting hydraulic flow efficiency, then what might be the evolutionary drivers of $\langle c\rangle$ variation between species? The fact that the ubiquitous GI measurements could be uncorrelated to measures of $3 \mathrm{D}$ connectivity (Fig. 9) means that, currently, the data needed to answer this question are unavailable. If, however, $\langle c\rangle$ is correlated with GI and air-seeding is the main way for embolisms to spread, then species such as those in the genus Acer might have to increase $\langle c\rangle$ at the expense of increasing vessel frequency. This is because GI and $\mathrm{VA}_{x}^{-1}$ are highly correlated in Acer (Lens et al., 2011). This would incur one of two costs: either there would be less space for 
Growth rings

(a)

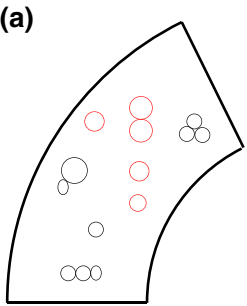

(b)

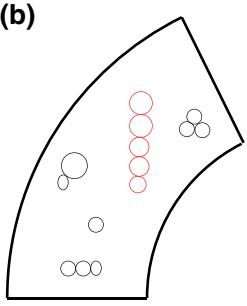

(c)

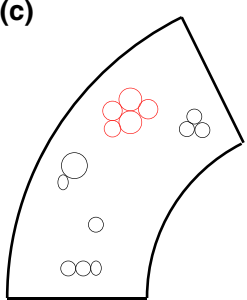

2D connectivity features
x-section connections of red vessels

$$
\begin{aligned}
& \text { \# of conduits: } 14 \\
& \text { \# of groups: } 8 \\
& \text { GI: } 14 / 8=1.75 \\
& \text { \# of x-section } \\
& \text { connections: } 14 \\
& <_{C_{\mathrm{x}}}>\text { : } 14 / 14=1
\end{aligned}
$$

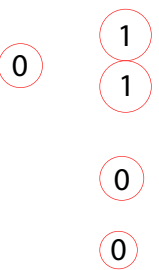

\# of conduits: 14

\# of groups: 5

GI: $14 / 5=2.8$

\# of x-section

connections: 20

$<c_{\mathrm{x}}>: 20 / 14 \approx 1.4$

\# of conduits: 14

\# of groups: 5

GI: $14 / 5=2.8$

\# of $x$-section

connections: 26

$<c_{x}>: 26 / 14 \approx 1.9$

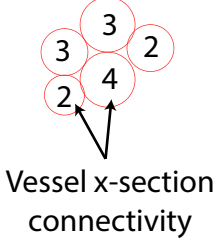

connectivity
Fig. 9 Illustration showing the discrepancy between the grouping index $(\mathrm{Gl})$ and transverse or cross-section ( $\mathrm{x}$-section) vessel connectivity $\left\langle c_{x}\right\rangle$. The $\mathrm{Gl}$ is defined as the number of vessels (number in green) divided by the number of vessel groups (number in orange). $\left\langle c_{x}\right\rangle$ is the number of cross-section vessel connections (number in purple) divided by the number of vessels in a transverse stem section. The vessels in black are invariant whereas those in red form different vessel multiples when comparing (a), (b) and (c). On the right-most column, the red vessels are highlighted and the cross-sectional connectivity langlec $c_{x}$ of each is written inside of it. The '\# of $x$-section connections' in the middle column is the sum of the vessel connectivities in the growth rings (left column) for both the black and red vessels. In particular when comparing (b) and (c), we see that one vessel group can have a higher connectivity with more tangential connections. While the illustrations in this figure are constrained to the cross-section, the same concepts apply in three dimensions with the 3D vessel connectivity property $\langle c\rangle$.

other types of cells in the xylem-like fibers and parenchyma, negatively affecting biomechanics and storage (Pratt \& Jacobsen, 2017); or the conduits would have smaller diameters to preserve space, with implications for hydraulic efficiency and construction costs in terms of carbon (Fig. S2). The latter is what is observed in the set of Acer species where a near-constant vessel packing fraction is conserved (Fig. S3; Lens et al., 2011). However, reliable, interfamily measurements of $\langle c\rangle$ are necessary to validate or correct this hypothesis.

Resistance to embolism spread in plant organs is determined by the intersection among multiple anatomical traits operating at different scales. The ultrastructure of pit membranes determines the size of the nanopores that both allow water flow and restrict embolism spread (Jansen et al., 2009; Lens et al., 2011; Li et al., 2016; Zhang et al., 2020). Wider vessels reduce water flow resistance but a larger surface area, with more pit membranes, has been suggested to facilitate embolism spread (Hargrave et al., 1994; Christman et al., 2009, 2012). A common theme among these traits, when they change, is that they affect $k_{x a}$ and $P_{50}$ in opposing ways. This reason is partly behind the expectation that safe species, with high resistance to drought, are less efficient with low hydraulic conductivity, and vice versa. This expectation leads to the hypothesis of the 'safety-efficiency' trade-off (Tyree et al., 1994) which has recently been shown to be weak (Gleason et al., 2016). However, the simulations perturbing vessel connectivity have shown that it has a significant effect on the slope of the VC (Figs 6, 7) regardless of changes in pit and vessel anatomy. These 'middle-scale' variations in xylem networks do not affect $P_{50}$ and $k_{x a \text {, max }}$, typical measures of xylem safety and efficiency, in clearly opposing ways (Manzoni et al., 2013). The weakness of the expected safety-efficiency trade-off among woody species (Gleason et al., 2016) might be because an increase in vessel connectivity, all else constant, could improve $P_{50}$ (to a certain extent; Fig. $6 \mathrm{~b}$ ) while not affecting $k_{x a, \max }$ (as observed through the $2 \mathrm{D}$ proxy GI; blue and gray scatter plots in Fig. 8), unlike how changes in other anatomical traits affect them.

'Middle-scale' variations encompass more than trends in vessel grouping, especially in flowering plans with vasicentric tracheids (Carlquist, 1984, 2009). In such species, much of the hydraulic connectivity between vessels is mediated by radial or tangential bands of conductive tracheids. As a result, such species with mostly solitary vessels will encounter different embolism spread dynamics. A recent study on three flowering species with mostly solitary vessels has concluded that solitary cavitation events dominate over events that involve groups of vessels (Johnson et al., 2020). Consequently, species possessing different patterns of vessel connectivity will not conform to similar $P_{50}$ and $k_{x a \text {, max }}$ trade-offs even if vessel and pit anatomy are identical.

In Acer, increasing conduit connectivity improves branch resistance to embolism spread without adversely affecting hydraulic conductivity. This may be true for all plants with vessels as the only water-conducting cell type such as Acer. In general, that means that when xylem network characteristics vary among woody species, the 'safety-efficiency' trade-off hypothesis applied to the segment level might not hold. This result was established using a combination of numerical simulations and theoretical tactics borrowed from network and percolation theory. Increasing average conduit connectivity invoked a trade-off between hydraulic pathway redundancy and embolism spread speed with respect to pressure. Pathway redundancy increased with conduit connectivity because conduit isolation was avoided. Above a critical conduit connectivity, improvements in redundancy were canceled by the speed of embolism spread due to a declining percolation threshold. These results underline the need to account for changes in average conduit connectivity, preferential arrangements and cell types among organs and species to successfully generalize hydraulic trends.

\section{Acknowledgements}

AM acknowledges funding from the National Science Foundation (NSF-AGS-1644382, NSF-AGS-2028633). AM, DMJ, 
DML and J-CD acknowledge the NSF for grant NSF-IOS1754893. The authors appreciate the critical feedback provided by Dr Gabriel G. Katul and Dr Frederic Lens.

\section{Author contributions}

AM planned and designed the research and developed the computational model and the theory. AM, DMJ, DML and J-CD analysed the data and model results and wrote the manuscript.

\section{ORCID}

Jean-Christophe Domec (D) https://orcid.org/0000-0003-04782559

Daniel M. Johnson (ID https://orcid.org/0000-0003-1015-9560

David M. Love (ID https://orcid.org/0000-0002-0582-6990

Assaad Mrad (DD https://orcid.org/0000-0003-4922-4446

\section{Data availability}

Branch anatomy data on the seven Acer species were obtained (Lens et al., 2011) after contacting the corresponding author of that study. The code for the model can be found at https:// github.com/mradassaad/Xylem_Network_Matlab.

\section{References}

Callaway DS, Newman MEJ, Strogatz SH, Watts DJ. 2000. Network robustness and fragility: percolation on random graphs. Physical Review Letters 85: 5468 5471.

Carlquist S. 1984. Vessel grouping in dicotyledon wood: significance and relationship to imperforate tracheary elements. Aliso: A Journal of Systematic and Evolutionary Botany 10: 505-525.

Carlquist S. 2009. Non-random vessel distribution in woods: patterns, modes, diversity, correlations. Aliso: A Journal of Systematic and Evolutionary Botany 27: $39-58$.

Choat B, Cobb AR, Jansen S. 2008. Structure and function of bordered pits: new discoveries and impacts on whole-plant hydraulic function. New Phytologist 177: 608-626.

Christman MA, Sperry JS, Adler FR. 2009. Testing the 'rare pit' hypothesis for xylem cavitation resistance in three species of Acer. New Phytologist 182: 664674.

Christman MA, Sperry JS, Smith DD. 2012. Rare pits, large vessels and extreme vulnerability to cavitation in a ring-porous tree species. New Phytologist 193: 713-720.

Cochard H, Cruiziat P, Tyree MT. 1992. Use of positive pressures to establish vulnerability curves: further support for the air-seeding hypothesis and implications for pressure-volume analysis. Plant Physiology 100: 205209.

Cohen R, Erez K, Ben-Avraham D, Havlin S. 2000. Resilience of the internet to random breakdowns. Physical Review Letters 85: 4626-4628.

Dixon HH, Joly J. 1895. On the ascent of sap. Philosophical Transactions of the Royal Society of London. Series B: Biological Sciences 186: 563-576.

Domec J-C, Gartner BL. 2001. Cavitation and water storage capacity in bole xylem segments of mature and young Douglas-fir trees. Trees 15: 204-214.

Ewers FW, Ewers JM, Jacobsen AL, López-Portillo J. 2007. Vessel redundancy: modeling safety in numbers. IAWA Journal 28: 373-388.

Gleason SM, Westoby M, Jansen S, Choat B, Hacke UG, Pratt RB, Bhaskar R, Brodribb TJ, Bucci SJ, Cao K-F et al. 2016. Weak tradeoff between xylem safety and xylem-specific hydraulic efficiency across the world's woody plant species. New Phytologist 209: 123-136.
Hacke UG, Jacobsen AL, Pratt RB. 2009. Xylem function of arid-land shrubs from California, USA: an ecological and evolutionary analysis. Plant, Cell \& Environment 32: 1324-1333.

Hacke UG, Sperry JS, Wheeler JK, Castro L. 2006. Scaling of angiosperm xylem structure with safety and efficiency. Tree Physiology 26: 689-701.

Hargrave KR, Kolb KJ, Ewers FW, Davis SD. 1994. Conduit diameter and drought-induced embolism in Salvia mellifera Greene (Labiatae). New Phytologist 126: 695-705.

Hölttä T, Vesala T, Nikinmaa E. 2007. A model of bubble growth leading to xylem conduit embolism. Journal of Theoretical Biology 249: 111-123.

Jacobsen AL, Pratt RB. 2018. Going with the flow: structural determinants of vascular tissue transport efficiency and safety. Plant, Cell \& Environment 41: 2715-2717.

Jansen S, Choat B, Pletsers A. 2009. Morphological variation of intervessel pit membranes and implications to xylem function in angiosperms. American Journal of Botany 96: 409-419.

Johnson KM, Brodersen C, Carins-Murphy MR, Choat B, Brodribb TJ. 2020. Xylem embolism spreads by single-conduit events in three dry forest angiosperm stems. Plant Physiology 184: 212-222.

Kaack L, Altaner CM, Carmesin C, Diaz A, Holler M, Kranz C, Neusser G, Odstrcil M, Schenk HJ, Schmidt V et al. 2019. Function and threedimensional structure of intervessel pit membranes in angiosperms: a review. IAWA Journal 40: 673-702.

Kanduč M, Schneck E, Loche P, Jansen S, Schenk HJ, Netz RR. 2020. Cavitation in lipid bilayers poses strict negative pressure stability limit in biological liquids. Proceedings of the National Academy of Sciences, USA 117: 10733-10739.

Konrad W, Katul G, Roth-Nebelsick A, Jensen KH. 2019. Xylem functioning, dysfunction and repair: a physical perspective and implications for phloem transport. Tree Physiology 39: 243-261.

Konrad W, Roth-Nebelsick A. 2003. The dynamics of gas bubbles in conduits of vascular plants and implications for embolism repair. Journal of Theoretical Biology 224: 43-61.

Lemaire C, Quilichini Y, Brunel-Michac N, Santini J, Berti L, Cartailler J, Conchon P, Badel É, Herbette S. 2021. Plasticity of the xylem vulnerability to embolism in Populus tremula $\mathrm{x}$ alba relies on pit quantity properties rather than on pit structure. Tree Physiology tpab018. doi: 10.1093/treephys/tpab018.

Lens F, Sperry JS, Christman MA, Choat B, Rabaey D, Jansen S. 2011. Testing hypotheses that link wood anatomy to cavitation resistance and hydraulic conductivity in the genus Acer. New Phytologist 190: 709-723.

Li S, Lens F, Espino S, Karimi Z, Klepsch M, Schenk HJ, Schmitt M, Schuldt B, Jansen S. 2016. Intervessel pit membrane thickness as a key determinant of embolism resistance in angiosperm xylem. IAWA Journal 37: 152-171.

Loepfe L, Martinez-Vilalta J, Pinol J, Mencuccini M. 2007. The relevance of xylem network structure for plant hydraulic efficiency and safety. Journal of Theoretical Biology 247: 788-803.

Manzoni S, Vico G, Katul G, Palmroth S, Jackson RB, Porporato A. 2013. Hydraulic limits on maximum plant transpiration and the emergence of the safety-efficiency trade-off. New Phytologist 198: 169-178.

Martínez-Vilalta J, Mencuccini M, Álvarez X, Camacho J, Loepfe L, Piñol J. 2012. Spatial distribution and packing of xylem conduits. American Journal of Botany 99: 1189-1196.

Mrad A, Domec J-C, Huang C-W, Lens F, Katul G. 2018. A network model links wood anatomy to xylem tissue hydraulic behaviour and vulnerability to cavitation. Plant, Cell \& Environment 41: 2718-2730.

Newman MEJ. 2018. Networks. Oxford, UK: Oxford University Press.

Pratt RB, Jacobsen AL. 2017. Conflicting demands on angiosperm xylem: tradeoffs among storage, transport and biomechanics. Plant, Cell \& Environment 40: 897-913.

Pratt RB, Jacobsen AL. 2018. Identifying which conduits are moving water in woody plants: a new HRCT-based method. Tree Physiology 38: 1200-1212.

Pratt RB, Percolla MI, Jacobsen AL. 2015. Integrative xylem analysis of Chaparral Shrubs. In: Hacke U, ed. Functional and ecological xylem anatomy. Cham, Switzerland: Springer International, 189-207.

Roth-Nebelsick A. 2019. It's contagious: calculation and analysis of xylem vulnerability to embolism by a mechanistic approach based on epidemic modeling. Trees 33: 1519-1533. 
Salleo S, Hinckley TM, Kikuta SB, Lo Gullo MA, Weilgony P, Yoon T-M, Richter H. 1992. A method for inducing xylem emboli in situ: experiments with a field-grown tree. Plant, Cell \& Environment 15: 491-497.

Sano Y, Morris H, Shimada H, Ronse De Craene LP, Jansen S. 2011. Anatomical features associated with water transport in imperforate tracheary elements of vessel-bearing angiosperms. Annals of Botany 107: 953-964.

Schenk HJ, Espino S, Romo DM, Nima N, Do AYT, Michaud JM, Papahadjopoulos-Sternberg B, Yang J, Zuo YY, Steppe K et al. 2017. Xylem surfactants introduce a new element to the cohesion-tension theory. Plant Physiology 173: 1177-1196.

Schenk HJ, Steppe K, Jansen S. 2015. Nanobubbles: a new paradigm for airseeding in xylem. Trends in Plant Science 20: 199-205.

Sperry JS, Saliendra NZ. 1994. Intra-and inter-plant variation in xylem cavitation in Betula occidentalis. Plant, Cell \& Environment 17: 1233-1241.

Sperry JS, Tyree MT. 1988. Mechanism of water stress-induced xylem embolism. Plant Physiology 88: 581-587.

Tononi G, Sporns O, Edelman GM. 1999. Measures of degeneracy and redundancy in biological networks. Proceedings of the National Academy of Sciences, USA 96: 3257-3262.

Tyree MT, Davis SD, Cochard H. 1994. Biophysical perspectives of xylem evolution: is there a tradeoff of hydraulic efficiency for vulnerability to dysfunction? IAWA Journal 15: 335-360.

Tyree MT, Zimmermann MH. 2002. Xylem structure and the ascent of sap. Springer series in wood science. Berlin, Germany: Springer.

Venturas MD, Pratt RB, Jacobsen AL, Castro V, Fickle JC, Hacke UG. 2019. Direct comparison of four methods to construct xylem vulnerability curves: differences among techniques are linked to vessel network characteristics. Plant, Cell \& Environment 42: 2422-2436.

Venturas MD, Sperry JS, Hacke UG. 2017. Plant xylem hydraulics: what we understand, current research, and future challenges. Journal of Integrative Plant Biology 59: 356-389.
Zhang Ya, Carmesin C, Kaack L, Klepsch MM, Kotowska M, Matei T, Schenk HJ, Weber M, Walther P, Schmidt V et al. 2020. High porosity with tiny pore constrictions and unbending pathways characterize the $3 \mathrm{D}$ structure of intervessel pit membranes in angiosperm xylem. Plant, Cell \& Environment 43: 1 .

\section{Supporting Information}

Additional Supporting Information may be found online in the Supporting Information section at the end of the article.

Fig. S1 The probability of tangential intervessel connections does not explain variation in $\mathrm{k}_{\mathrm{xa}, \max }$ and $\mathrm{P}_{50}$.

Fig. S2 The effect of $\mathrm{VA}_{\mathrm{x}}^{-1}$ on GI and $c$.

Fig. S3 The seven Acer species maintain a tight relationship between $\mathrm{VA}_{\mathrm{x}}^{-1}$ and average vessel lumen area.

Methods S1 Modeling xylem networks: sap flow.

Methods S2 Calculating GI and $\mathrm{VA}_{\mathrm{x}}^{-1}$ in the model.

Please note: Wiley Blackwell are not responsible for the content or functionality of any Supporting Information supplied by the authors. Any queries (other than missing material) should be directed to the New Phytologist Central Office.

\section{About New Phytologist}

- New Phytologist is an electronic (online-only) journal owned by the New Phytologist Foundation, a not-for-profit organization dedicated to the promotion of plant science, facilitating projects from symposia to free access for our Tansley reviews and Tansley insights.

- Regular papers, Letters, Viewpoints, Research reviews, Rapid reports and both Modelling/Theory and Methods papers are encouraged. We are committed to rapid processing, from online submission through to publication 'as ready' via Early View our average time to decision is $<26$ days. There are no page or colour charges and a PDF version will be provided for each article.

- The journal is available online at Wiley Online Library. Visit www.newphytologist.com to search the articles and register for table of contents email alerts.

- If you have any questions, do get in touch with Central Office (np-centraloffice@lancaster.ac.uk) or, if it is more convenient, our USA Office (np-usaoffice@lancaster.ac.uk)

- For submission instructions, subscription and all the latest information visit www.newphytologist.com 